Alexander Blokh, Department of Mathematics, University of Alabama in Birmingham, University Station, Birmingham, AL 35294-2060. Current address: Mathematisches Institut, Universtät Erlangen-Nürnberg, Erlangen 91054, Germany. e-mail: ablokhmath.uab.edu

\title{
DENSITY OF PERIODIC ORBITS IN $\omega$-LIMIT SETS WITH THE HAUSDORFF METRIC
}

\begin{abstract}
We prove that if $f$ is a continuous interval map such that all wandering intervals converge to periodic orbits, then the family of periodic orbits is dense in $\omega$-limit sets with Hausdorff metric.
\end{abstract}

\section{Introduction}

Periodic orbits together with their various characteristics are rather important for dynamical systems. In the case of an interval map $f$ it is reflected by the fact that periodic points are dense in some sets which are important for the dynamics of $f$ (from now on by $f$ we always denote a continuous interval map). The first result in this direction is due to A. N. Sharkovsky who proved in [S1] that the set of periodic points is dense in the set of all recurrent points of $f$ (see also $[\mathrm{N}]$ ). Since recurrent points are dense in the center of a dynamical system it shows that, on the interval the center of a dynamical system coincides with the closure of the set of periodic points. A related result was later obtained in [B] where it was proven that if $\mu$ is an $f$-invariant probabilistic measure, then a point $x$ with $\mu(\omega(x))=1$ exists if and only if $\mu$ can be approximated by measures concentrated on periodic orbits; in particular any ergodic measure is approximated by a measure concentrated on a periodic orbit.

\footnotetext{
Key Words: Interval maps, $\omega$-limit sets, periodic points, Hausdorff metric

Mathematical Reviews subject classification: 54H20, 58F03, 58F08

Received by the editors December 8, 1997

*The author was partially supported by NSF Grant DMS 9626303 and Humboldt Foun-
} dation. 
Another interesting topic in one-dimensional dynamics is studying of $\omega$ limit sets with Hausdorff metric on them. More precisely, let $\mathcal{K}$ be the class of compact subsets of $[0,1]$ endowed with Hausdorff metric $H$ and let $\mathcal{Z}(f) \subset \mathcal{K}$ be the family of all $\omega$-limit sets of an interval map $f$. There has been a series of papers studying the family $\mathcal{Z}(f)$ and related topics. In particular the properties of the map $\omega_{f}:[0,1] \rightarrow \mathcal{Z}(f)$ defined by $\omega_{f}: x \rightarrow \omega_{f}(x)$ were studied in $[\mathrm{BC}]$. It was shown that this map is almost never continuous but is always in the second Baire class, some general theorems relating the Baire class of $\omega_{f}$ to its Borel class and to certain notions of semi-continuity of $\omega_{f}$ were also obtained.

One more paper where the properties of $\mathcal{Z}(f)$ are studied is [BBHS] where it is proved that $\mathcal{Z}(f)$ is compact which in the light of the essential discontinuity of $\omega_{f}$ seems somewhat surprising. A useful technical result obtained in [BBHS] is a criterion connecting a local behavior of a map in a small neighborhood of a given compact set $A$ with the property of the set $A$ to be the $\omega$-limit set of some point; the compactness of $\mathcal{Z}(f)$ was obtained in [BBHS] as a corollary of this criterion (see also [BS]).

The problem we study in this paper is whether or not periodic orbits are dense in the family $\mathcal{Z}(f)$. One can consider this problem as a natural continuation of the above mentioned ones ([S1], [B], [BBHS]). The question however significantly differs from the old ones. For example, it is easy to see that the results of $[\mathrm{B}]$ do not apply to the situation in question; indeed, in $[\mathrm{B}]$ we deal with measures while our problem concerns $\omega$-limit sets. Yet some tools developed in $[\mathrm{B}]$ prove to be useful for us as we shall see later.

The difference between the situation for measures and $\omega$-limit sets can be seen from the fact that actually periodic orbits are not always dense in the family $\mathcal{Z}(f)$ (we shall give an example later in the paper). However there is a natural and from the practical point of view the most interesting class of interval maps for which periodic orbits are dense in $\mathcal{Z}(f)$. Let us introduce this class of maps. Let $I$ be an interval such that $I, f(I), \ldots$ are pairwise disjoint; then we call $I$, wandering. Denote by $|X|$ the Lebesgue measure of a set $X$; in particular, if $I$ is an interval, then $|I|$ is its length. It is clear that if $I$ is wandering, then $\left|f^{n}(I)\right| \rightarrow 0$. Therefore all points $x \in I$ have the same $\omega$-limit set which we denote by $\omega(I)$.

We consider a class of maps $\mathcal{G}$ such that for any $f \in \mathcal{G}$ if $I$ is a wandering interval, then $\omega(I)$ is a periodic orbit. Even making only this assumption about the maps (i.e. without assuming piecewise monotonicity) one can establish their nice properties. In particular, for maps $f \in \mathcal{G}$ it is possible to describe their typical in topological sense limit behavior (see [B]): if $f \in \mathcal{G}$, then there is a massive set $E \subset[0,1]$ such that for any $x \in E$ the set $\omega(x)$ is either a 
periodic orbit, or a limit set of special kind called, solenoid or, generalized adding machine (see definitions later) or a finite union of pairwise disjoint closed intervals cyclically permuted by $f$ on which $f$ is transitive. However - and from the point of view of applications more importantly - it also turns out that smooth interval maps belong to $\mathcal{G}$ (see [L], [BL], [MMS] for details). This justifies our interest to the maps from the class $\mathcal{G}$, and now we can state our

Main Theorem. Let $f \in \mathcal{G}$. Then the family of all periodic orbits of $f$ is dense in $\mathcal{Z}(f)$.

This result seems to be reasonably sharp; in fact below we give an easy example of a piecewise monotone (in fact bimodal) interval map $g \notin \mathcal{G}$ for which the conclusion of Main Theorem fails. To do so we will need a well known in one-dimensional dynamics construction of 'gluing in' an interval with all its preimages which essentially allows us to represent discontinuous interval maps as continuous interval maps on appropriately chosen invariant subsets. This can be done for a wide variety of discontinuous interval maps, yet for our purposes it is enough to work with the following specific case.

Consider a discontinuous map $\tau:[0,1] \rightarrow[0,1]$ defined as $\tau(x)=x+$ $\theta \bmod 1$ where $0<\theta<1$ is an irrational number; in other words, $\tau$ is a discontinuous lifting of an irrational rotation by $\theta$ onto interval. Obviously the only point of discontinuity of $\tau$ is $a=1-\theta$.

Let us now construct a new map $f$ by means of 'blowing up' the point $a$. That is, let us look at $a$ as if it were a small interval, say, $\left[a^{\prime}, b^{\prime}\right]$, on which the map $f$ is defined as follows:

(a) points $a^{\prime \prime}, b^{\prime \prime}$ are chosen so that $a^{\prime}<a^{\prime \prime}<b^{\prime \prime}<b^{\prime}$;

(b) $f\left[a^{\prime}, a^{\prime \prime}\right]=1, f\left[b^{\prime \prime}, b^{\prime}\right]=0, f \mid\left[a^{\prime \prime}, b^{\prime \prime}\right]$ is linear.

The next step is to consider the first $\tau$-preimage $\tilde{a}$ of $a$ and also replace it by an interval, say, $\left[a_{1}^{\prime}, b_{1}^{\prime}\right]$ on which our new map $f$ is defined as monotone and such that $a_{1}^{\prime}, b_{1}^{\prime}$ are mapped into the points $a^{\prime}, b^{\prime}$ appropriately (this depends on whether $\tau$ reverses monotonicity at $\tilde{a}$ or not). Clearly one can repeat this construction over and over; carefully choosing the lengths of 'inserted' intervals one will get in the end a limit map $f$ with the following properties.

(1) $f:[c, d] \rightarrow[c, d]$ is a continuous map;

(2) There are points $a^{\prime}<a^{\prime \prime}<b^{\prime \prime}<b^{\prime}$ such that $f \mid\left[c, a^{\prime}\right]$ is strictly increasing, $f\left[a^{\prime}, a^{\prime \prime}\right]=d, f \mid\left[a^{\prime \prime}, b^{\prime \prime}\right]$ is strictly decreasing, $f\left[b^{\prime \prime}, b^{\prime}\right]=c, f \mid\left[b^{\prime}, d\right]$ is strictly increasing. 
(3) If $A=\left\{x: \operatorname{orb}_{f}(x) \in\left[c, a^{\prime}\right] \cup\left[b^{\prime}, d\right]\right\}$, then $A$ is in fact a minimal set on which $f$ is monotonically at most 2-to- 1 semiconjugate to $\tau$ everywhere so that there are no periodic orbits contained in $\left[c, a^{\prime}\right] \cup\left[b^{\prime}, d\right]$; in particular, $c, d \in A$.

It is easy to see that in fact no periodic orbit approximates $A$. Indeed, let $\varepsilon=\min \left(\left|a^{\prime \prime}-a^{\prime}\right|,\left|b^{\prime \prime}-b^{\prime}\right|\right)$. If a periodic orbit $Q$ is such that $H(Q, A)<\varepsilon$, then $Q \subset\left[c, a^{\prime \prime}\right] \cup\left[b^{\prime \prime}, d\right]$. Due to the property (3) of the map $f$ the set $Q$ cannot belong to $\left[c, a^{\prime}\right] \cup\left[b^{\prime}, d\right]$. Therefore $Q \cap\left(\left[a^{\prime}, a^{\prime \prime}\right] \cup\left[b^{\prime \prime}, b^{\prime}\right]\right)$ is not empty. However by the construction it implies that either $d \in Q$ or $c \in Q$ which is impossible.

Actually, it is easy to construct the map $f$ so that essentially the same properties hold with the only difference that $f$ will have no flat spots (a , flat spot is a maximal non-degenerate interval on which a map is a constant); in order to do this we will have to define $f$ on $\left[a^{\prime}, a^{\prime \prime}\right]$ and $\left[b^{\prime \prime}, b^{\prime}\right]$ more carefully so that both intervals will be wandering with orbits imitating orbits of points 1 and 0 under $\tau$ which in turn will imply for $f$ the same conclusions as before. We will not dwell on these details assuming that enough motivation for Main Theorem has already been provided.

Acknowledgments I would like to thank Andy Bruckner, Paul Humke and Jaroslav Smital the work with whom on the paper [BBHS] was not just a starting point for the present paper but also a great pleasure. I also would like to thank G. Keller for inviting me to Erlangen and useful discussions as well as Erlangen University for its hospitality.

\section{Preliminaries}

In our study we rely upon some results of [B] which we now describe. Let $I$ be an interval such that $I, f(I), \ldots, f^{n-1}(I)$ are pairwise disjoint while $f^{n}(I)=I$. Then $I$ is called a, periodic interval and both the union $\bigcup_{i=0}^{n-1} f^{i}(I)$ and the family of intervals $\left\{f^{i}(I)\right\}_{i=0}^{n-1}=$ orb $I$ are called a , cycle of intervals. Also, a continuous map defined on a finite union of pairwise disjoint intervals and cyclically permuting these intervals is called, non-strictly periodic; for instance, a map $f$ restricted to a cycle of intervals is non-strictly periodic. Now, let $I_{0} \supset I_{1} \supset \ldots$ be periodic intervals with periods $m_{0}, m_{1}, \ldots$ Obviously $m_{i+1}$ is a multiple of $m_{i}$ for all $i$. If $m_{i} \rightarrow \infty$, then the intervals $\left\{I_{j}\right\}_{j=0}^{\infty}$ are said to be, generating and any invariant closed set $S \subset Q=\bigcap_{j \geq 0}$ orb $I_{j}$ is called a, solenoidal set; if $Q$ is nowhere dense, then we call $Q$ a , solenoid. It turns out that solenoidal $\omega$-limit sets are one of the three major classes of $\omega$-limit sets existing for interval maps. 
To introduce another class of $\omega$-limit sets let us suppose that a map $f$ has a cycle of intervals $M=I \cup f(I) \cup \cdots \cup f^{n-1}(I)$. Consider a set $\{x \in M$ : for any relative neighborhood $U$ of $x$ in $M$ we have $\overline{\operatorname{orb} U}=M\}$; it is easy to see that this is a closed invariant set. It is called a , basic set and denoted by $B(M, f)$ provided it is infinite. Basic sets are components of the decomposition of the set $\overline{\operatorname{Per} f}$ constructed in [B]. Their properties are listed in Theorem 2.1, however first we need one more definition. Let $F: M \rightarrow M$ and $G: K \rightarrow K$ be two non-strictly periodic maps of the same period, $\phi: M \rightarrow K$ be a (non-strict) monotone semiconjugacy between $F$ and $G$ and $B \subset M$ be a $F$-invariant closed subset of $M$. If $\phi(B)=J$ and for any $x \in J$ we have $\phi^{-1}(x) \cap B=\partial \phi^{-1}(x)$, then we say that $\phi$, almost conjugates $F \mid B$ to $G$. Here $\partial Z$ is the , boundary of a set $Z$. Clearly this is equivalent to the fact that closures of intervals complementary to $B$ in $M$ are exactly flat spots of $\phi$ (and therefore $B$ is exactly the set of points which do not have $\phi$-'flat spot' neighborhoods).

Now we list the properties of basic sets.

Theorem 2.1. ([B]) Let $f$ be a continuous interval map, $A=\omega(x)$. Then one of the following statements holds for $A$.

(1) A is a periodic orbit, maximal by inclusion among $\omega$-limit sets.

(2) $A$ is a solenoidal set.

(3) There exists a basic set $B$ such that $A \subset B$.

Moreover, the following additional statements hold.

(a) Basic sets are perfect maximal by inclusion $\omega$-limit sets.

(b) Distinct basic sets intersect each other at finite or empty sets, no three basic sets have a common point and any basic set is disjoint from any solenoidal set.

(c) If $B=B(K, f)$ is a basic set of period $m$, then there exists a transitive non-strictly periodic map $g$ of the same period and a map $\phi$ which almost conjugates $f \mid K$ and $g$.

(d) If $\omega(x)$ belongs to a basic set $B$, then there exists a point $x^{\prime} \in B$ such that $\omega(x)=\omega\left(x^{\prime}\right)$.

This result justifies the approach to some problems in one-dimensional dynamics which is based on considering the cases (1), (2) and (3) from Theorem 2.1 separately and then combining the results into one. In particular, this 
allows us to establish some properties of invariant measures of interval maps which prompted the problem in question. Note that while studying the cases (1) and (2) is more or less straightforward, that of the case (3) usually consists of two parts:

(a) working with non-strictly periodic transitive maps;

(b) lifting the results to an $\omega$-limit set contained in a basic set.

For the part (a) the following facts about transitive maps are useful.

Lemma 2.2. ([B]) Let $g:[0,1] \rightarrow[0,1]$ be a transitive map. Then one of the following statements holds.

(1) $g$ is mixing.

(2) There exists a fixed point $a \in(0,1)$ such that $g[0, a]=[a, 1], g[a, 1]=$ $[0, a]$ and both $g^{2} \mid[0, a]$ and $g^{2}[a, 1]$ are mixing.

The following lemma deals with expanding properties of mixing maps.

Lemma 2.3. ([B]) Let $f:[0,1] \rightarrow[0,1]$ be mixing. Then for any $\delta>0$ and any open $U$ there exists $N=N(\delta, U)$ such that $f^{n}(U) \supset[\delta, 1-\delta]$ for any $n \geq N$.

Corollary 2.4 easily follows from Lemmas 2.2 and 2.3 .

Corollary 2.4. Let $f:[0,1] \rightarrow[0,1]$ be a transitive map. Then there exists $\varepsilon^{\prime}(f)=\varepsilon^{\prime}>0$ such that for any $\delta>0$ and $0<\varepsilon<\varepsilon^{\prime}$ the following holds.

1. There exists a number $n=n(\delta, \varepsilon)$ such that for any interval $U$ of the length greater than $\delta$ we have $\left|f^{m}(U)\right| \geq \varepsilon$ for any $m \geq n$. (In particular if $x \in U$, then for any such $m$ the distance between $f^{m}(x)$ and at least one of the endpoints of $f^{m}(U)$ is greater than $\varepsilon / 2$.)

2. Periodic points are dense in $[0,1]$.

Proof. (1) If $f$ is mixing, then let $\varepsilon^{\prime}=1$. If $f$ is not mixing then by Lemma 2.2 there exists $a \in(0,1)$ such that $f[0, a]=[a, 1]$ and $f[a, 1]=[0, a]$. In this case let $\varepsilon^{\prime}=\min (a, 1-a)$. Let $\varepsilon<\varepsilon^{\prime}$.

We will need the following construction. Clearly there exists a finite family of intervals $U_{1}, \ldots, U_{l}$ of the length, say, $\delta / 2$ such that any interval of the length $\delta$ contains at least one of them. Let us now consider mixing and non-mixing cases separately.

Let $f$ be mixing. Applying Lemma 2.3 to each $U_{i}$ and the interval $I=$ $[(1-\varepsilon) / 4,(3+\varepsilon) / 4]$ of the length $(\varepsilon+1) / 2>\varepsilon$ we see that $f^{r}\left(U_{i}\right) \supset I$ for 
sufficiently big $r$ and any $i$. Therefore for any interval $W$ of the length bigger than $\delta$ and any sufficiently big $r$ we have $\left|f^{r}(W)\right|>\varepsilon$ which proves statement (1) for mixing maps.

Suppose that $f$ is not mixing. Then there is a point $a \in(0,1)$ such that $f[0, a]=[a, 1], f[a, 1]=[0, a]$ and both $f^{2} \mid[0, a]$ and $f^{2} \mid[a, 1]$ are mixing. Choose closed intervals $J^{\prime} \subset(0, a)$ and $J^{\prime \prime} \subset(a, 1)$ of the length $\left(\varepsilon+\varepsilon^{\prime}\right) / 2$. Let $U_{i} \subset[0, a]$. Then by Lemma 2.3 there exists $p_{i}$ such that $f^{2 r}\left(U_{i}\right) \supset J^{\prime}$ for $r \geq p_{i}$. On the other hand $f\left(U_{i}\right)$ is a non-degenerate interval; so by Lemma 2.3 there exists $q$ such that $f^{2 r}\left(f\left(U_{i}\right)\right)=f^{2 r+1}\left(U_{i}\right) \supset J^{\prime \prime}$ for $r \geq q_{i}$. Therefore for sufficiently big $m$ we have $\left|f^{m}\left(U_{i}\right)\right|>\varepsilon$. Similarly one can consider the case when $U_{i} \subset[a, 1]$ as well as the case when $a \in \operatorname{int} U_{i}$. This completes the proof of the first part of the statement (1). The second part follows immediately.

(2) Due to Lemma 2.3 for any $x \in(0,1), \varepsilon>0$ there is a closed interval $I \subset[x-\varepsilon, x+\varepsilon]$ and a number $N$ such that $f^{N}(I) \supset I$. So there is a periodic point in $I$ which completes the proof.

We call the constant $\varepsilon^{\prime}(f)$ from Corollary 2.4 the, expansiveness of $f$.

The following two lemmas are of technical nature and will be useful in the proof of Main Theorem.

Lemma 2.5. Let $F: X \rightarrow X$ be a continuous map of a compact metric space $(X, d)$ and let $M=\omega(x)$ have the following property: there are subsets $M_{0}, \ldots, M_{n-1}$ of $M$ such that $M=\bigcup_{k=0}^{n-1} M_{k}, M_{i+1}=f\left(M_{i}\right), 0 \leq i \leq k-2$ and $M_{0}=f\left(M_{k-1}\right)$ (so that all $M_{i}$ are $F^{n}$-invariant). Then if for any $\varepsilon$ there is an $F^{n}$-periodic orbit $Q$ with $H\left(Q, M_{0}\right)<\varepsilon$, then for any $\delta$ there is an $f$-periodic orbit $P$ with $H(P, M)<\delta$.

Proof. An $F^{n}$-periodic orbit $Q$ is $\varepsilon$-close to $M_{0}$ in Hausdorff metric if for any point $x \in Q$ there is a point $y \in M_{0}$ such that $d(x, y)<\varepsilon$ and for any $z \in M_{0}$ there is $\zeta \in Q$ such that $d(z, \zeta)<\varepsilon$. Let us choose $\varepsilon$ so that for any two points $u, v$ if $d(u, v)<\varepsilon$ then $d\left(f^{i}(u), f^{i}(v)\right)<\delta$ for any $0 \leq i \leq n$.

Let $Q=\operatorname{orb}_{F^{n}}(w)$ be an $F^{n}$-periodic orbit such that $H\left(Q, M_{0}\right)<\varepsilon$ and $P=\operatorname{orb}_{F}(w)$ be the corresponding $F$-periodic orbit. Let us prove that $H(P, M)<\delta$. Indeed, if $x \in M$ then there exists a point $x^{\prime} \in M_{0}$ and a number $j, 0 \leq j<n$ such that $F^{j}\left(x^{\prime}\right)=x$. By the assumption there is a point $y^{\prime} \in Q$ such that $d\left(x^{\prime}, y^{\prime}\right)<\varepsilon$. Therefore by the choice of $\varepsilon$ we have $d\left(F^{j}\left(x^{\prime}\right), F^{j}\left(y^{\prime}\right)\right)<\delta$; in other words there is a point $F^{j}\left(y^{\prime}\right) \in P$ in the $\delta$-neighborhood of $F^{j}\left(x^{\prime}\right)=x$.

It remains to prove that for any $z \in P$ there is $\zeta \in M$ such that $d(z, \zeta)<$ $\delta$. Indeed, if $z \in P$ then there exists $z^{\prime} \in Q$ and $j, 0 \leq j<n$ such that $F^{j}\left(z^{\prime}\right)=z$. Now, there exists $\zeta^{\prime} \in M_{0}$ such that $d\left(z^{\prime}, \zeta^{\prime}\right)<\varepsilon$ which implies that $d\left(F^{j}\left(z^{\prime}\right), F^{j}\left(\zeta^{\prime}\right)\right)=d\left(z, F^{j}\left(\zeta^{\prime}\right)\right)<\delta$ and completes the proof. 
For any map $F$ let $S_{n}^{n+k}(x, F)=\left\{F^{n}(x), F^{n+1}(x), \ldots, F^{n+k}(x)\right\}$.

Lemma 2.6. Let $F: X \rightarrow X$ be a continuous map of a compact metric space $X$ into itself and let $k \in \mathbb{N}$. Suppose $\omega(x)=M$ be infinite and $A \subset M$ is finite. Then there exists a neighborhood $U \supset A$ for which one can find an $n$ so large that $S_{n}^{n+k}(x, F)$ is disjoint from $U$.

Proof. Consider the finite set $B=\bigcup_{i=0}^{k} F^{i}(A)$ and choose a neighborhood $W$ of $B$ such that $M \not \subset W$. Then choose a neighborhood $U$ of $A$ such that $F^{i}(U) \subset W$ for any $0 \leq i \leq k$. Let us prove that $U$ has the required property. Indeed, by the choice of $W$ there is an arbitrarily big $N=n+k$ such that $F^{N}(x) \notin W$. Then $F^{n+j}(x) \notin U$ for any $0 \leq j \leq k$ since otherwise $f^{N}(x) \in$ $F^{k-j}(U) \subset W$ which is a contradiction. Clearly since $N$ is arbitrarily large, $n=N-k$ is also arbitrarily large which completes the proof.

Finally, we will also need the following well known fact (see, e.g. [S2]).

Lemma 2.7. ([S2]) If $\omega(x)$ is finite then it is a periodic orbit.

\section{Density of Periodic Orbits in $\omega$-Limit Sets}

We begin our study of the density of periodic orbits in the family of $\omega$-limit sets with the Hausdorff metric by considering this question for transitive interval maps and then for subsets of basic sets. This approach is justified by Theorem 2.1 and Lemma 2.5. However for transitive interval maps we prove a refined version of the Main Theorem.

First we need an important technical lemma describing the dynamics of a point $x$ with infinite limit set $\omega(x)=A$ in small neighborhoods of a periodic point $a \in A$. It will be convenient to denote the minimal distance between a point $z$ and a compact set $K$ by $d(z, K)$.

Lemma 3.1. Let $x$ be a point whose $\omega$-limit set $\omega(x)=A$ is infinite. Let $a \in A$ be a periodic point of period $m$. Then for any $\varepsilon>0$ there exist open neighborhoods $U \supset V \ni a$ and points $y, z \in A \cap U$ distinct from a such that

1. $|U|<\varepsilon ;$

2. if $f^{j}(x) \in V$ for some $j$ and $k$ is the least number such that $f^{j+m k}(x) \notin$ $U$, then any interval connecting $f^{j+m k}(x)$ and any point from $V$ contains at least one of the points $y, z$.

Proof. Let $\varepsilon>0$ is given. It is well known that periodic points are not isolated in limit sets containing them. Consider two cases according to whether $a$ is not isolated in $A$ from one side or from two sides. 
a) Suppose that $a$ is not isolated in $A$ from either side. Choose $U$ so that $|U|<\varepsilon$ and there are points $y, z$ of $A$ in both components of $U \backslash\{a\}$. Choose a neighborhood $V$ of $a$ so that $V \subset(y, z)$. Then the required follows immediately because no matter on what side of $a$ the point $f^{j+m k}(x)$ lies any interval connecting $f^{j+m k}(x)$ and any point from $V$ contains at least one of the points $y, z$.

b) Suppose that $a$ is isolated from the right. Choose $U=(b, c)$ so that $|U|<\varepsilon$ and the following additional properties hold:

1. $(a, c) \cap A=\emptyset$ and $d(c, A)=|c-a|$;

2. $f^{m}(b, a)$ lies to the left of $c$.

Now, choose a neighborhood $V^{\prime}=(u, v) \subset U$ of $a$ so that:

1. $f^{m}\left(V^{\prime}\right) \subset U$;

2. $|v-a| \leq|c-a| / 2$

3. $(b, u) \cap A \neq \emptyset$.

Then choose $V \subset V^{\prime}$ so small, that the least number $k$ with $f^{k}(x) \in V$ is very big; so big that for all $i \geq k$ we have $d\left(f^{i}(x), A\right)<|v-a|$. Let us show that this implies that for all $i \geq k$ the points $f^{i}(x)$ avoid the interval $[v, c)$. Indeed, by the choice of the distances for any point $\zeta \in[v, c)$ we have $d(\zeta, A) \geq|v-a|$ while by the assumption for all $i \geq k$ we have $d\left(f^{i}(x), A\right)<|v-a|$.

Choose a point $y=z \in(b, u) \cap A$. Let $j$ be a number such that $f^{j}(x) \in$ $V$ and let $k$ be the smallest number such that $f^{j+m k}(x) \notin U$. Then $x^{\prime}=$ $f^{j+m k-m}(x) \in U$ and we consider now the question of where in $U$ can the point $x^{\prime}$ lie. We already know that $x^{\prime} \notin(v, c)$. On the other hand $x^{\prime} \notin V^{\prime}$ since $f^{m}\left(V^{\prime}\right) \subset U$ while $f^{m}\left(x^{\prime}\right) \notin U$. So the only possibility is that $x^{\prime} \in(b, u)$. However $f(b, a)$ lies to the left of $c$; therefore so does $f^{m}\left(x^{\prime}\right)$. We conclude that $f^{j+m k}(x)=f^{m}\left(x^{\prime}\right) \leq b$ and thus indeed any interval connecting $f^{j+m k}(x)$ and any point from $V$ contains $y=z$. This completes the proof.

The next step is to introduce an important technical notion of fine collections of intervals. Let $U=U_{1} \cup U_{2} \cup \cdots \cup U_{n}$ be the union of closed intervals such that $A=\omega(x) \subset U$. We consider a specific class of such unions. Namely, $U$ is called an $\varepsilon$-fine collection of intervals with respect to the set $A$ or simply a fine collection if the following holds.

1. Intervals in $U$ are non-degenerate, their interiors are pairwise disjoint, all intervals are non-disjoint from $A$ and have the lengths less than $\varepsilon$. 
2. Suppose that $[a, b]$ is one of the intervals from $U$. Then neither $a$ nor $b$ is contained in the orbit of $x$. Moreover, if $a \in A(b \in A)$ then $\omega(a)(\omega(b))$ and $a(b)$ is approximated from within $[a, b]$ by points of the orbit of $x$. On the other hand if $a \notin A$ then $f(a) \notin$ int $U_{1} \cup$ int $U_{2} \cup \cdots \cup \operatorname{int} U_{n}$ and the same holds for $b$.

3. $\operatorname{orb}_{f}(x) \subset U$.

There are obvious properties of the endpoints of intervals $\left\{U_{i}\right\}$ that follow immediately from the definition of a fine collection. Before we state them we introduce a few special sets. Namely, let $\Pi$ be the set of all endpoints of intervals from $U$ and let also $\Pi^{\prime}=\Pi \cap A, \Pi^{\prime \prime}=\Pi \backslash A$; clearly $\Pi, \Pi^{\prime}$ and $\Pi^{\prime \prime}$ are finite. Also, by part (3) of the definition of a fine collection, for any $j$ there exists $s(j)$ such that $f^{j}(x) \in U_{s(j)}$. We shall see shortly that if $\omega(x)=A$ is infinite then $s(j)$ is well-defined.

Lemma 3.2. For any $k$ there exist a number $\rho^{\prime}>0$ such that if $\left|f^{m}(x)-a\right|<$ $\rho^{\prime}$ for an endpoint $a$ of the interval $U_{s(m)}$ then $a \in \Pi$ and points $f^{i+m}(x), f^{i}(a)$ belong to the same interval $U_{s(i+m)}$ for all $0 \leq i \leq k$.

Proof. First of all we may assume $\rho^{\prime}$ to be so small that $d(b, \operatorname{orb}(x))>\rho^{\prime}$ for any $b \in \Pi^{\prime \prime}$. Thus if $\left|f^{m}(x)-a\right|<\rho^{\prime}$ then $a \in \Pi^{\prime}$.

Now, if $a \in \Pi^{\prime}$, then the definition implies that $\operatorname{orb}_{f}(a) \subset U$. For any $0 \leq i \leq k$ the distance between $f^{i}(a)$ and all the intervals $\left\{U_{j}\right\}$ which do not contain $f^{i}(a)$ is positive. Let $\rho$ be the minimum of all these distances and choose $\rho^{\prime}$ so small that $|u-v|<\rho^{\prime}$ implies that $\left|f^{i}(u)-f^{i}(v)\right|<\rho, 0 \leq i \leq k$. Therefore, if $\left|f^{m}(x)-a\right|<\rho^{\prime}$ then $\left|f^{m+i}(x)-f^{i}(a)\right|<\rho$. On the other hand $f^{m+i}(x) \in U_{s(m+i)}$. If $f^{i}(a) \notin U_{s(m+i)}$ then by the choice of $\rho$ we must have $\left|f^{m+i}(x)-f^{i}(a)\right|>\rho$, a contradiction. So $f^{i}(a) \notin U_{s(m+i)}$ which completes the proof.

The following lemma immediately follows from the definition.

Lemma 3.3. Points of $\Pi^{\prime \prime}$ are mapped into $\Pi$ or outside $U$; so eventually all points of $\Pi^{\prime \prime}$ are mapped either outside $U$ or into $\Pi^{\prime}$.

Lemma 3.4 relies upon Lemma 3.3.

Lemma 3.4. If $A$ is infinite then the point $x$ is never mapped into a point from $\Pi$ (and so the interval $U_{s(i)}$ containing $f^{i}(x)$ is well defined).

Proof. Since $A$ is infinite, $x$ is never mapped into a point from $\Pi^{\prime}$. On the other hand if it is mapped into $\Pi^{\prime \prime}$ then by Lemma 3.3 it is eventually mapped into $\Pi^{\prime}$ (which impossible as we have just seen) or outside $U$ (which is impossible by property (3) of fine collections). This competes the proof. 
From now on we fix a transitive map $f:[0,1] \rightarrow[0,1]$ and assume that $\varepsilon^{\prime}(f)=\varepsilon^{\prime}$ is the expansiveness of $f$. Moreover, suppose that $A=\omega(x) \subset U$ is an infinite limit set and $U=U_{1} \cup U_{2} \cup \cdots \cup U_{n} \supset A$ is an $\varepsilon^{\prime} / 2$-fine collection with respect to $A$. Moreover, let $\Gamma$ be the set of all points from those periodic orbits to which points from $\Pi^{\prime}$ converge. Also, a closed interval with endpoints $a, b$ is denoted by $[a ; b]$ (where both possibilities $a<b$ and $a>b$ are included).

We now prove the central technical lemma of this section. Here speaking of semineighborhoods we always mean compact semineighborhoods.

Lemma 3.5. Let $f:[0,1] \rightarrow[0,1]$ be transitive and let $\varepsilon^{\prime}(f)=\varepsilon^{\prime}$ be the expansiveness of $f$. Suppose that $A=\omega(x) \subset U$ is an infinite limit set, $U=$ $U_{1} \cup U_{2} \cup \cdots \cup U_{n} \supset A$ is an $\varepsilon^{\prime} / 2$-fine collection with respect to $A$. Then there exists a number $\alpha>0$ such that in any semineighborhood $W$ of $x$ there exists an interval $V$ such that for some integer $t$ we have $f^{j}(V) \subset U_{s(j)}, 0 \leq j \leq t$ and $f^{t}\left(V_{t}\right)$ contains a point $\zeta^{\prime} \in A$ with its $\alpha$-neighborhood.

Proof. Let us describe the following process. For the sake of definiteness let $x \in W=V_{0} \subset U_{s(1)}$ be a left semineighborhood of $x$. We construct an interval $V_{1}$ as follows. We know that $f(x) \in \operatorname{int}\left(U_{s(1)}\right)$. If $f\left(V_{0}\right) \subset U_{s(1)}$, we set $V_{1}=V_{0}$. Otherwise considering the family of all left semineighborhoods of $x$ we can get the biggest with the $f$-image in $U_{s(1)}$ and denote it by $V_{1}$. Obviously then $G=[a ; f(x)] \subset f\left(V_{1}\right) \subset U_{s(1)}$ for an endpoint $a$ of $U_{s(1)}$ and $a \in \Pi$.

Clearly, similarly we can construct by induction a nested sequence of left semineighborhoods of $x$ such that $V_{0} \supset V_{1} \supset \ldots$, and the construction is well defined. More precisely, let $f^{m}(x) \in \operatorname{int} U_{s(m)}$. If $f^{m+1}\left(V_{m}\right)$ is contained in $U_{s(m)}$, we set $V_{m+1}=V_{m}$. Otherwise we again consider the family of left semineighborhoods of $x$, contained in $V_{m}$, get the biggest with the $f^{m+1}$ image in $U_{s(i)}$ and denote it by $V_{m+1}$. Then for an appropriate interval $G=$ $\left[a ; f^{m+1}(x)\right]$ with $a$ being an endpoint of $U_{s(m+1)}(a \in \Pi)$, we have $G \subset$ $f^{m+1}\left(V_{m+1}\right) \subset U_{s(m+1)}$.

We have $R=\bigcap_{i=0}^{\infty} V_{i}=\{x\}$. Indeed, otherwise $R$ is a closed interval whose images are contained in the intervals $U_{j}, 1 \leq j \leq n$ and therefore have length smaller than $\varepsilon^{\prime} / 2$ all the time which contradicts Corollary 2.4 and the choice of $\varepsilon^{\prime}$. This implies that the process in question eventually does not depend on the choice of $W=V_{0}$. More precisely, let $V_{0} \supset V_{0}^{\prime}$ be two left semineighborhoods of $x$ and let $V_{i}$ and $V_{i}^{\prime}$ denote the $i$-th semineighborhoods of $x$ constructed for $V_{0}$ and $V_{0}^{\prime}$ respectively. Then there exist the least $k$ such that $V_{k} \varsubsetneqq V_{0}^{\prime}$ and the least $i$ such that $V_{i}^{\prime} \varsubsetneqq V_{0}^{\prime}$. It is easy to see then that $i \leq k, V_{i}^{\prime}=V_{k}$ and, moreover, for all $r \geq 0$ we have $V_{i+r}^{\prime}=V_{k+r}$. Therefore the arguments we are about to apply to some $V_{0}$ in fact apply to all $V_{0}$, so that the constant $\alpha$ which we find for this $V_{0}$ in fact is independent of $V_{0}$. 
So, let us fix $V_{0}$. Also, let us denote the set of those $m$ for which $f^{m}\left(V_{m}\right) \supset$ $\left[d_{m} ; f^{m}(x)\right], d_{m} \in \Pi$, by $\mathbf{A}$; to avoid ambiguity with the choice of $d_{m}$ in the case when $f^{m}\left(V_{m}\right)=U_{s(m)}$ we always choose $d_{m}$ to be the left endpoint of $U_{s(m)}$ (which actually does not make any difference). Consider a few cases concerning various types of behavior of a point $d_{m}$.

Suppose first that for all sufficiently big $m \in \mathbf{A}$ and some $\delta^{\prime}>0$ we have $\left|f^{m}\left(V_{m}\right)\right|>\delta^{\prime}$. (In particular, this holds if $\left|f^{m}(x)-d_{m}\right|>\delta^{\prime}$.) We may assume that $\delta^{\prime}<\varepsilon^{\prime} / 2$. Then by Corollary 2.4 and because all interval $U_{j}$ are shorter than $\varepsilon^{\prime} / 2$, there exists a number $K$ such that for any $K$ consecutive and sufficiently big integers there exists an element of $\mathbf{A}$ among them. Indeed, if $\left|f^{m}\left(V_{m}\right)\right|>\delta^{\prime}$ then by Corollary $2.4\left|f^{n\left(\delta^{\prime}, \varepsilon^{\prime} / 2\right)}\left(f^{m}\left(V_{m}\right)\right)\right|>\varepsilon^{\prime} / 2$ and so some number $i \leq n\left(\delta^{\prime}, \varepsilon^{\prime} / 2\right)$ must belong to $\mathbf{A}$. Now it suffices to set $K=n\left(\delta^{\prime}, \varepsilon^{\prime} / 2\right)$.

Clearly this implies that for some $\delta^{\prime \prime}<\delta^{\prime}$ we have that $\left|f^{m}\left(V_{m}\right)\right|>\delta^{\prime \prime}$ for any $m$. Indeed, transitivity implies that there are no flat spots of $f$. For any $x$ define a function $\Phi(x)=\min _{0 \leq i \leq K}\left\{\left|f^{i}\left[x, x+\delta^{\prime}\right]\right|\right\}$. Then $\Phi(x)$ is a continuous positive function which therefore has a positive minimal value $\delta^{\prime \prime}$, and we may assume that $\delta^{\prime \prime}<\delta^{\prime}$. Now, for any sufficiently large $j$ there exists $m \in \mathbf{A}$ such that $m \leq j<m+K$. Then by the construction we have $V_{j}=V_{m}$ and $\left|f^{j}\left(V_{j}\right)\right|=\left|f^{j-m}\left(f^{m}\left(V_{m}\right)\right)\right| \geq \delta^{\prime \prime}$ because $\left|f^{m}\left(V_{m}\right)\right| \geq \delta^{\prime}$.

Let us show how this implies what is required. Indeed, since $A$ is infinite, there exists a non-isolated point $z \in A$. We may assume that $z$ is non-isolated in $A$ from the right. Then we can find three points $z^{\prime}, z^{\prime \prime}, z^{\prime \prime \prime} \in A$ such that $z<z^{\prime}<z^{\prime \prime}<z^{\prime \prime \prime}<z+\delta^{\prime \prime} / 2$. Let

$$
0<\alpha<1 / 2\left(\min \left\{\left|z^{\prime}-z\right|,\left|z^{\prime \prime}-z^{\prime}\right|,\left|z^{\prime \prime \prime}-z^{\prime \prime}\right|,\left|z+\delta^{\prime \prime} / 2-z^{\prime \prime \prime}\right|\right\}\right) .
$$

Suppose that for some sufficiently big $i$ we have $\left|f^{i}(x)-z^{\prime \prime}\right|<\alpha$. Since $\left|f^{i}\left(V_{i}\right)\right|>\delta^{\prime \prime}$, at least one component among the two into which $f^{i}(x)$ divides $f^{i}\left(V_{i}\right)$ is longer than $\delta^{\prime \prime} / 2$. If its the left component, then it contains $z^{\prime}$ with its $\alpha$-neighborhood and if its the right component, then it contains $z^{\prime \prime \prime}$ with its $\alpha$-neighborhood. In other words we were able to find a constant $\alpha(f)=\alpha>0$ with the property that there exists an arbitrary interval $V_{i}$ close to $x$ such that $f^{i}\left(V_{i}\right)$ contains a point from $A$ with its $\alpha$-neighborhood and $f^{j}\left(V_{i}\right)$ is contained in one of the intervals from $U$ for $0 \leq j \leq i$.

So we may assume that there are arbitrarily big numbers $m \in \mathbf{A}$ for which $\left|f^{m}\left(V_{m}\right)\right|$ (and therefore $\left.\left|f^{m}(x)-d_{m}\right|\right)$ is arbitrarily small. Choosing a subsequence we may assume that in fact there is a point $d \in \Pi$ such that $f^{m}(x) \rightarrow d$ and $\left[d ; f^{m}(x)\right] \subset f^{m}\left(V_{m}\right)$; moreover, if there are periodic or periodic points which appear infinitely many times as points $d_{m}, m \in \mathbf{A}$, then we choose $d$ as one of them; that is, $d$ neither periodic nor periodic only if there are no periodic or periodic points appearing infinitely many times among the points 
$d_{m}, m \in \mathbf{A}$. Notice that by Lemma $3.4 x$ is never mapped into $\Pi$ and so $f^{m}(x) \neq d$ for any $m \in \mathbf{A}$. Moreover, the set $\Pi^{\prime \prime}$ is disjoint from $\omega(x)$; so $d \in \omega(x)=A$. Consider some cases.

1) The point $d$ is periodic (of period, say, $l$ ). Let us show that then Lemma 3.1 implies what is required. Indeed, choose sufficiently small neighborhoods $U \supset V$ and points $y, z$ as guaranteed by Lemma 3.1. Then choose a large number $m \in \mathbf{A}$ so that $d_{m}=d, f^{m}(x) \in V$. Suppose that $k$ is the least number such that $f^{l k+m}(x) \notin U$. Then $\left(d ; f^{l k+m}(x)\right)$ contains either a point $y$ or a point $z$ with a neighborhood of fixed size. On the other hand by the construction $\left[d ; f^{l k+m}(x)\right] \subset f^{l k+m}\left(V_{l k+m}\right)$. Clearly, this implies the required.

2) The point $d$ is periodic. In this case it is enough to map $d$ by the corresponding power of $f$ into a periodic point and then apply the arguments from the case 1 ).

3) The point $d$ is neither periodic nor periodic. Since $d \in \Pi^{\prime}$, we see that $d$ converges to a periodic orbit of a point from the set $\Gamma$, say, of period $l$. Let $u$ be a point from this periodic orbit. Choose sufficiently small neighborhoods $U \supset$ $V$ of $u$ and corresponding points $y, z$ as guaranteed by Lemma 3.1. Also, choose a much smaller neighborhood $V^{\prime}$ of $u$ so that $V \supset V^{\prime}$ and let $\varepsilon$ be smaller than the length of either of components of $V^{\prime} \backslash\{u\}$. Choose $k$ sufficiently large so that for all $j \geq 0$ we have $\left|f^{k+j}(d)-f^{j}(u)\right|<\varepsilon$. Then choose $\delta$ so small that $\left|f^{s}(d)-f^{s}(v)\right|<\varepsilon, 0 \leq s \leq k$ for any point $v$ such that $|d-v|<\delta$. Finally, choose $m$ so big that $\left|d-f^{m}(x)\right|<\delta$. Then $\left|f^{s}(d)-f^{m+s}(x)\right|<\varepsilon$ for all $0 \leq s \leq k$. Moreover, by the choice of $k$ the further images of $d$ stay in $\varepsilon$-neighborhood of the orbit of $u$ while the orbit of $x$ must leave $U$ because the limit set of $x$ is infinite. We can again apply Lemma 3.1 which, similarly to the cases 1) and 2), implies the existence of the required number $\alpha$. This completes the proof of the lemma.

Lemma 3.6 relies upon Lemma 3.5.

Lemma 3.6. Let $f:[0,1] \rightarrow[0,1]$ be transitive, let $A=\omega(x) \subset U$ be an infinite limit set where $U=U_{1} \cup U_{2} \cup \cdots \cup U_{n}$ is an $\varepsilon^{\prime}(f) / 2$-fine with respect to $A$ collection. Then there is a periodic orbit $Q \subset \bigcup_{i=1}^{n} \operatorname{int} U_{i}$ which visits interiors of all intervals $U_{i}, 1 \leq i \leq n$.

Proof. Let $\alpha>0$ be a constant from Lemma 3.6. Consider the orbit of $x$ and choose numbers $N<M$ in the following way.

First of all, we require that the set $S_{0}^{N}(x, f)$ intersect all intervals from $U$ and the same holds for $S_{N+1}^{N+M}(x, f)$. Also, if some of the endpoints of intervals from $U$ are periodic then let $M$ be greater than all their periods. Finally, let $N$ and $M$ be such that $H\left(S_{0}^{N}(x, f), A\right)<\alpha / 4, H\left(S_{N+1}^{N+M}(x, f), A\right)<\alpha / 4$. (To get these last properties we may need to replace $x$ by its forward iterate under 
sufficiently high power of $f$.) Choose a small neighborhood $W$ of $x$ so that for any $0 \leq j \leq N+M$ we have $f^{j}(W) \subset$ int $U_{s(j)}$. (This is possible since by Lemma 3.4 the orbit of $x$ does not pass through endpoints of the intervals from $U$.) Notice that together with the choice of $n, M$ this implies that sets $f^{i}(W), 0 \leq i \leq N$ visit all intervals from $U$ and also sets $f^{i}(W), N+1 \leq i \leq M$ visit all intervals from $U$. Moreover, we may assume that $\left|f^{j}(W)\right|<\alpha / 4$ for any $0 \leq j \leq N+M$.

By Lemma 3.5 there is a number $t$ and an interval $V \subset W$ such that $f^{i}(V) \subset U_{s(i)}, 0 \leq i \leq t$ and $f^{t}(V)$ contains a point $\zeta^{\prime} \in A$ with its $\alpha$ neighborhood. Notice that by the choice of $W$ we have $t>N+M$. Now, since $H\left(S_{0}^{N}(x, f), A\right)<\alpha / 4$, there exists $l \leq N$ such that $f^{l}(x)$ is $\alpha / 4$-close to $\zeta^{\prime}$. Thus the $\alpha / 4$-neighborhood of $f^{l}(x)$ is contained in $f^{t}(V)$; on the other hand by the choice of $W$ we see that $f^{l}(V)$ is contained in the $\alpha / 4$ neighborhood of $f^{l}(x)$ which implies that $f^{t}(V) \supset f^{l}(V)$. Therefore there is a periodic point $z \in f^{l}(V)$ such that $f^{t-l}(z)=z$. By the choice of $V$ and $W$ we see that orb $z \subset \bigcup_{i=1}^{n}$ int $U_{i}$. Indeed, the fact that orb $z \subset U$ is obvious. Suppose that $z$ is mapped into an endpoint $y$ of one of intervals from $U$ by some iterate of $f$; this endpoint is then periodic of period less than $M$. The period in question is in fact the period of $z$ as well which implies that the point $y$ can be found among points $f^{N+1}(z), f^{N+2}(z), \ldots, f^{N+M}(z)$. On the other hand by the choice of $W$ we have that $f^{j}(W)$ is contained in the interior of some interval from $U$ for $N \leq j \leq N+M$. This contradiction implies that orb $z \subset \bigcup_{i=1}^{n}$ int $U_{i}$. To complete the proof it remains to notice that sets $f^{i}(V), N \leq i \leq M$ visit all intervals from $U$ because they are contained in $f^{i}(W)$, and that $l \leq N<N+M \leq t$.

Now we are ready to prove our Main Theorem. Recall, that we consider a class of maps $\mathcal{G}$ such that for any $f \in \mathcal{G}$ if $I$ is a wandering interval then $\omega(I)$ is a periodic orbit. Also, by $\mathcal{K}$ we denote the class of compact subsets of $[0,1]$ endowed with Hausdorff metric $H$ and by $\mathcal{Z}(f) \subset \mathcal{K}$ we denote the family of all $\omega$-limit sets of an interval map $f$.

Main Theorem. Let $f \in \mathcal{G}$. Then the family of all periodic orbits of $f$ is dense in $\mathcal{Z}(f)$.

Proof. Let $A=\omega(x)$; our aim is to find a periodic orbit $Q$ such that $H(Q, A)$ is arbitrary small. This is trivial if $A$ is a periodic orbit itself. Since by Lemma 2.7 all finite limit sets are periodic orbits, we may assume from now on that $A$ is infinite.

Suppose that $A$ is a solenoidal set. Then the fact that all wandering intervals of $f$ converge to periodic orbits implies that in fact $A$ is a solenoid. Indeed, let $V \supset V_{1} \supset \ldots$ be a nested sequence of periodic intervals of periods 
$m_{0}, m_{1}, \ldots, m_{i} \rightarrow \infty$, generating $A$. If $A$ is not a solenoid then there is a non-trivial component of $Q=\bigcap_{i=0}^{\infty} \operatorname{orb}_{f}\left(V_{i}\right)$; in other words, there exists a sequence $r_{0} \leq r_{2} \leq \ldots$ and a non-degenerate interval $J=\bigcap_{i=0}^{\infty} f^{r_{i}}\left(V_{i}\right)$. Clearly $J$ is a wandering interval which does not converge to a periodic orbit; this contradiction implies that $A=Q=\bigcap_{i=0}^{\infty} \operatorname{orb}_{f}\left(V_{i}\right)$ is a solenoid. Therefore $\varepsilon_{i}=\max \left\{\left|f^{r}\left(V_{i}\right)\right|: 0 \leq i \leq m_{i}-1\right\}$ converges to 0 while $i$ tends to infinity. On the other hand for any $i$ there is a periodic orbit $Q_{i} \subset \operatorname{orb}_{f}\left(V_{i}\right)$ and it follows from the definition that $H\left(Q_{i}, A\right) \leq \varepsilon_{i}$. So to find a periodic orbit which is no more than $\varepsilon$-distant from $Q$ in the sense of Hausdorff metric it is enough to take $Q_{i}$ with $\varepsilon_{i}<\varepsilon$.

It remains now to consider the case of an infinite limit set $A$ contained in a basic set. We prove this in a few steps establishing along the way some properties of basic sets and transitive maps.

Property A. If $g:[0,1] \rightarrow[0,1]$ is mixing then for any $\varepsilon$ there is a periodic orbit $Q$ such that $H(Q,[0,1])<\varepsilon$.

Proof of Property A. Let us make use of Lemma 2.3. Indeed, we can find a finite family of pairwise disjoint closed intervals $U_{1}, \ldots, U_{n}$ such that $R=\bigcup_{i=1}^{n} U_{i} \subset(0,1)$ and the length of any of these intervals and any of the gaps between them is less than $\varepsilon / 3$. Then due to Lemma 2.3 there is a number $N$ such that $f^{N}\left(U_{i}\right) \supset R$ for any $1 \leq i \leq n$. Hence there is a periodic point $z$ such that $z \in U_{1}, f^{N}(z) \in U_{2}, \ldots, f^{N(n-1)}(z) \in U_{n}, f^{N n}(z)=z$. The choice of the intervals $U_{i}, 1 \leq i \leq n$ implies now that $H\left(\operatorname{orb}_{f}(z),[0,1]\right)<\varepsilon$.

Property A implies Property B.

Property B. Let $A$ have a non-empty interior. Then for any $\varepsilon$ there is a periodic orbit $Q$ such that $H(Q, A)<\varepsilon$.

Proof of Property B. It is easy to see that if $A$ has a non-empty interior then in fact $A$ is a cycle of intervals on which $f$ is transitive. Let $A=\bigcup_{i=0}^{k-1} f^{k}(I)$ be the cycle of intervals of period $k$ where $I=[c, d]$. Then by Lemma 3.2 either $f^{k} \mid I$ is mixing or there is a point $a \in I$ such that $f^{k}[c, a]=[a, d], f^{k}[a, d]=[c, a]$ and both $f^{2 k} \mid[c, a]$ and $f^{2 k} \mid[a, d]$ are mixing. In any case, there is an interval $J$ and a number $m$ such that $\bigcup_{i=0}^{m-1} f^{i}(J)=A$, any two iterates of $J$ either coincide or have at most one point in common and $f^{m} \mid J$ is mixing (either $J=[c, d], m=k$ or $[c, a], m=2 k$ ). By Lemma 2.5 it is enough to show that $f^{m} \mid J$ has the property that $J$ as a limit set of $f^{m}$ is approximated in the sense of Hausdorff metric by periodic orbits; this follows from Property A.

From now on we assume that $A=\omega(x) \subset B=B(K), B$ is a basic set and that $A$ is nowhere dense. Let $K$ be a cycle of intervals of period $k$ and let $I$ be 
one of the intervals from $K$. By Theorem 2.1 we may also assume that $x \in B$. Denote $A \cap f^{i}(I)$ by $A_{i}$ for any $0 \leq i \leq k-1$. Then $f\left(A_{i}\right)=A_{i+1}(0 \leq i \leq$ $k-2), f\left(A_{k-1}\right)=A_{0}$. Let $f^{n}=F$; by Lemma 2.5 it is enough to show that $A_{0}$ can be arbitrary well approximated by an $F$-periodic orbit. By Theorem $2.1 F(I)=I, F \mid I$ is monotonically semiconjugate by a map $\phi$ to a transitive interval map $g:[0,1] \rightarrow[0,1]$. Also, we can assume that $x \in B_{0}=B \cap I$. Our aim is to find for any $\varepsilon>0$ an $F$-periodic orbit $Q$ so that $H\left(Q, A_{0}\right)<\varepsilon$; so from now on we consider the map $F$ restricted to $I$ instead of original map $f$.

We need to establish a few more technical properties. If $H$ is a closed set then we call an open interval $W$ an, $H$-gap if $W$ is complementary to $H$.

Property C. If $y$ is a point of a B-gap whose limit set is contained to B (e.g., if $y$ is an endpoint of the gap) then it converges to a periodic orbit.

Proof of Property C. Due to the existence of the monotone semiconjugacy between $F \mid I$ and a map $g$ the gap in question is either eventually mapped into a periodic $B_{0}$-gap or wandering and thus converging to a periodic orbit. In either case the assumption that $\omega(y) \subset B$ implies that $\omega(y)$ is a periodic orbit.

Property D. Suppose $y \in B_{0}$ is a limit point for $B_{0}$ from a side $T$ but is not a limit point of $A_{0}$ from the side $T$; then in any small $T$-semineighborhood of $y$ there is an F-periodic point $y^{\prime}$ such that $\phi\left(\operatorname{orb}_{F}\left(y^{\prime}\right)\right)$ is disjoint from $\phi\left(A_{0}\right)=C_{0}$.

Proof of Property D. If $U$ is a $T$-semineighborhood of $y$ then $\phi(U)$ is nondegenerate by Theorem 2.1. Since $g$ is transitive, by Corollary $2.4 g$-periodic points are dense in $[0,1]$. Thus we can find a $g$-periodic point $z$ in int $\phi(U)$. The monotonicity of $\phi$ now implies that the $\phi$-preimage of $z$ contains an $F$-periodic point which we denote by $y^{\prime}$. Since $z \notin C_{0}$, we see that $\phi\left(\operatorname{orb}_{F}\left(y^{\prime}\right)\right)=\operatorname{orb}_{g}(z)$ is disjoint from $C_{0}$. Notice that Property D (unlike Property C) does not depend on the assumptions about $f$.

Let us now prove the Main Theorem. Consider first the case when $A_{0}=B_{0}$. Clearly the continuity of $\phi$ implies that there is $\delta>0$ such that $|\phi[x-\varepsilon, x+\varepsilon]| \geq$ $\delta$ for any $x \in B_{0}$. Also, by Property A we can find a $g$-periodic orbit $Q$ such that $H(Q,[0,1])<\delta / 3$. Then there is an $F$-periodic orbit $P$ such that $\phi(P)=Q$ (we may even choose $P \subset B$ ). Let us show that $H\left(P, B_{0}\right)<\varepsilon$. Indeed, otherwise there exists a point $x \in B_{0}$ such that $[x-\varepsilon, x+\varepsilon] \cap P=\emptyset$. On the other hand $|\phi[x-\varepsilon, x+\varepsilon]| \geq \delta$ which implies that there is a point $z \in$ $\operatorname{int}(\phi[x-\varepsilon, x+\varepsilon]) \cap Q$. Then $\phi^{-1}(z) \subset[x-\varepsilon, x+\varepsilon]$ and so $[x-\varepsilon, x+\varepsilon] \cap P \neq \emptyset$. The contradiction implies that $H\left(P, B_{0}\right)=H\left(P, A_{0}\right) \leq \varepsilon$. 
So from now on we assume that $A_{0} \neq B_{0}$, or, equivalently, $\phi\left(A_{0}\right)=C_{0}$ is nowhere dense in $[0,1]$ (and thus $A_{0}$ is nowhere dense in $B_{0}$ ). We continue by constructing a finite family of closed intervals with pairwise disjoint interiors $U=U_{1} \cup \cdots \cup U_{n} \supset A_{0}$ with some additional properties. First, we may assume that $\varepsilon>0$ is sufficiently small so that for any $x, y$ such that $|x-y|<\varepsilon$ we have $|\phi(x)-\phi(y)|<\varepsilon^{\prime}(g) / 2$. In what follows we construct a finite set $V$ of points some of which will be the endpoints of our future intervals $U_{i}$. We begin by assuming that $V=\emptyset$ and will be adding points to this set.

Let $u_{0}$ be the leftmost point of $A_{0}$. Suppose first that $u_{0}$ is a limit point of the set $B_{0}$ from the left. Then by Property $\mathrm{D}$ there is an $f$-periodic point $v_{0} \in\left(u_{0}-\varepsilon / 4, u_{0}\right)$ such that $\phi\left(\operatorname{orb}_{F}\left(v_{0}\right)\right)$ is a $g$-periodic orbit disjoint from $C_{0}$. We add $\operatorname{orb}_{F}\left(v_{0}\right)$ to the set $V$. Suppose now that $u_{0}$ is the right endpoint of a $B_{0}$-gap. By Property $\mathrm{C}$ we may assume that $\operatorname{orb}_{F}(x)$ never enters this $B_{0}$-gap; in this case let $v_{0}=u_{0}$ and add the point $v_{0}$ to the set $V$.

Consider now a few possibilities for the interval $\left(u_{0}+\varepsilon / 4, u_{0}+\varepsilon / 2\right)=W$. First suppose that $W$ is not disjoint from $B_{0}$. Then because $A_{0}$ is nowhere dense in $B_{0}$ we can find a point $v^{\prime} \in\left(W \cap B_{0}\right) \backslash A_{0}$. By Property $\mathrm{D}$ there is an $F$-periodic point $v_{1} \in W$ such that $\phi\left(\operatorname{orb}_{F}\left(v_{1}\right)\right)$ is disjoint from $C_{0}$; in this case we add the entire $\operatorname{orb}_{F}\left(v_{1}\right)$ to the set $V$ and set $v_{1}^{\prime}=v_{1}$.

Now suppose that $W$ is contained in a $B_{0}$-gap, say, $(a, b)$. If $a \notin A_{0}$ we can by Property $\mathrm{D}$ find a periodic point $v_{1}<a$ close to $a$ so that $\left[v_{1}, a\right] \cap A_{0}=\emptyset$ and $\phi\left(\operatorname{orb}_{F}\left(v_{1}\right)\right)$ is disjoint from $C_{0}$. Then we $\operatorname{add}_{\operatorname{orb}_{F}}\left(v_{1}\right)$ to $V$. On the other hand if $a \in A_{0}$ then we set $v_{1}=a$ and add $v_{1}$ to $V$. In any case we also set $v_{1}^{\prime}=b$. Notice that in this case (i.e. when $W$ is contained in a $B_{0}$-gap) the sets $\omega\left(v_{1}\right)$ and $\omega\left(v_{1}^{\prime}\right)$ are periodic orbits (by the construction and by Property C).

To go on we need to establish some properties of our construction. First of all, one of the following holds.

(1) $u_{0}-\varepsilon / 4<v_{0} \leq u_{0}<u_{0}+\varepsilon / 4<v_{1}=v_{1}^{\prime}<u_{0}+\varepsilon / 4$. (This corresponds to the case when $W$ is non-disjoint from $B_{0}$.) or

(2) $u_{0}-\varepsilon / 4<v_{0} \leq u_{0}<v_{1} \leq u_{0}+\varepsilon / 4<u_{0}+\varepsilon / 2 \leq v_{1}^{\prime}$. (This corresponds to the case when $W$ is disjoint from $B_{0}$.)

Let us now make a few remarks concerning our construction.

a) Points which do not belong to $A$ are all periodic and added to the set $V$ together with their orbits while points from $A$ no matter whether periodic or not are added to the set $V$ 'individually', i.e. without adding the entire periodic orbit. 
b) Although the point $v_{1}^{\prime}$ takes part in the construction we do not include this point in the set $V$.

c) By Property $C$ in the case (2) the point $x$ cannot visit $W$. Thus, replacing $x$ by its appropriate forward image we may assume that in any case $\left[v_{1}, v_{1}^{\prime}\right]$ is disjoint from $\operatorname{orb}_{F}(x)$.

d) So far for a point $v \in V$ we have the following choices: a) $v \notin A_{0}$ is a periodic point such that $\phi\left(\operatorname{orb}_{F}(v)\right)$ is disjoint from $C_{0}$; b) $v \in A_{0}$, $\omega_{F}(v)$ is a periodic orbit and either $v=v_{0}=u_{0}$ is approximated by the orbit of $x$ only from the right, or $v=v_{1}=v_{1}^{\prime}$ is approximated by the orbit of $x$ from both sides, or $v=v_{1}<v_{1}^{\prime}$ is approximated by the orbit of $x$ only from the left and $\omega_{F}\left(v_{1}^{\prime}\right)$ is a periodic orbit.

Now, let us continue dealing with the set $A_{0}$ to the right of $v_{1}^{\prime}$. Denote the leftmost point of $A_{0} \cap\left[v_{1}^{\prime}, \infty\right)$ by $u_{1}$ and repeat our construction with some additions. Namely, if $v_{1}^{\prime} \notin A_{0}$ then we find $v_{2}$ and extend the set $V$ as before with only the additional property that $v_{1}^{\prime}<v_{2}$ which is clearly possible. If $v_{1}^{\prime} \in A_{0}$ then $v_{1}^{\prime}$ is the right endpoint of a $B$-gap approximated from the right by the orbit of $x$ in which case we set $v_{2}=v_{1}^{\prime}$ and add $v_{1}^{\prime}$ to $V$. It is clear that we can continue the construction until the entire set $A_{0}$ is covered, i.e. until for some $k$ we have $A_{0} \subset \bigcup_{i=0}^{k}\left[v_{2 i}, v_{2 i+1}\right]$; since for any $i$ we have $v_{2 i+1}^{\prime}-v_{2 i} \geq \varepsilon / 4$ the process will end in a finite number of steps.

Consider now the set of points $V$. They divide the whole interval into subintervals. Take only those among these subintervals which have infinite intersections with the orbit of $x$ and denote them by $U_{1}, \ldots, U_{m}$. Consider various possibilities which can realize for $U_{i}$.

1) $U_{i}=\left[d, d^{\prime}\right]$ and $d \notin A_{0}$. Then by the construction $d$ is an $F$-periodic point and $\phi\left(\operatorname{orb}_{F}(d)\right) \cap C_{0}=\emptyset$.

2) $U_{i}=\left[d, d^{\prime}\right]$ and $d \in A_{0}$. By the construction then $\omega_{F}(d)$ is a periodic orbit and $d$ is approached by $x$ from the right.

3) $U_{i}=\left[d, d^{\prime}\right]$ and $d^{\prime} \notin A_{0}$. Then by the construction $d^{\prime}$ is an $F$-periodic point and $\phi\left(\operatorname{orb}_{F}\left(d^{\prime}\right)\right) \cap C_{0}=\emptyset$.

4) $U_{i}=\left[d, d^{\prime}\right]$ and $d^{\prime} \in A_{0}$. By the construction then $\omega_{F}\left(d^{\prime}\right)$ is a periodic orbit and $d^{\prime}$ is approached by $x$ from the left.

Consider the family of intervals $\phi\left(U_{i}\right)=V_{i}$. Then it follows easily from the construction and properties 1) - 4) that they form an $\varepsilon^{\prime}(g) / 2$-fine collection. Therefore by Lemma 3.2 there is a $g$-periodic orbit $P$ which belongs to the set 
$\bigcup_{i=1}^{m} \operatorname{int} V_{i}$ and enters each $V_{i}$ at least once. If $Q$ is an $F$-periodic orbit such that $\phi(Q)=P$ then obviously $Q \in \bigcup_{i=1}^{m} \operatorname{int} U_{i}$ and $Q$ enters each $U_{i}$ at least once. Notice, that $\cup U_{i} \supset A_{0}$ and the length of each $U_{i}$ is less than $\varepsilon / 2$. This implies that $H\left(Q, A_{0}\right)<\varepsilon$ and completes the proof.

\section{References}

[B] A. Blokh, The Spectral Decomposition for One-Dimensional Maps, Dynamics Reported, 4 (1995), 1-59.

[BBHS] A. Blokh. A. Bruckner, P. Humke. J. Smital, On the metric space of $\omega$-limit sets of continuous maps of the interval, Trans. Amer. Math. Soc., 348 (1996), 1357-1372.

[BL] A. Blokh, M. Lyubich, Non-existence of Wandering Intervals and Structure of Topological Attractors of One-Dimensional Dynamical Systems.2 The Smooth Case, Erg. Th. and Dyn. Syst., 9 (1989) 751758.

[BC] A. Bruckner, J. Ceder, Chaos in terms of the map $x \rightarrow \omega(x, f)$, Pac. J. of Math., 156, No. 1 (1992), 63-96.

[BS] A. Bruckner, J. Smital, The structure of $\omega$-limit sets for continuous maps of the interval, Mathematica Bohemica, 117 (1992), 42-47.

[L] M. Lyubich, Non-existence of Wandering Intervals and Structure of Topological Attractors of One-Dimensional Dynamical Systems. 1 The Case of Negative Schwarzian, Erg. Th. and Dyn. Syst. 9 (1989), 737-749.

[MMS] M. Martens, W. de Melo, S. van Strien, Julia-Fatou-Sullivan Theory for Real One-Dimensional Dynamics, Acta Math., 168 (1992), 273318.

[N] Z. Nitecki, Periodic and limit orbits and the depth of the center for piecewise monotone interval maps, Proc. Amer. Math. Soc., 80 (1980), 511-514.

[S1] A. N. Sharkovsky, Non-wandering points and the center a continuous map of the line into itself, Dop. Acad. Nauk Ukr. RSR Ser. A (1964), $865-868$.

[S2] A. N. Sharkovsky, Continuous maps on the set of limit points of an iterated sequence, Ukr. Math. J., 18, No. 5 (1966), 127-130. 